\title{
Functional equations of the Gołąb-Schinzel type on a cone
}

\author{
J. Chudziak1 ${ }^{1}$ Z. Kočan ${ }^{2}$
}

Received: 25 August 2014 / Accepted: 8 June 2015 / Published online: 5 July 2015

(C) The Author(s) 2015. This article is published with open access at Springerlink.com

\begin{abstract}
We determine the solutions of conditional composite type functional equations related to some problems in mathematical meteorology and fluid mechanics.
\end{abstract}

Keywords Mathematical meteorology - Fluid mechanics · Gołąb-Schinzel type equations $\cdot$ Convex cone $\cdot$ Continuity on rays

Mathematics Subject Classification $\quad 39 B 12 \cdot 39$ B52

\section{Introduction}

Dealing with some problems in mathematical meteorology and fluid mechanics (e.g. evaporation of cloud droplets and water discharging from a reservoir) Kahlig and Matkowski [15] noted that the corresponding nonlinear differential equations exhibit some symmetries which can be expressed by the functional equation

$$
F\left(x+y s(x)^{r}\right)=s(x) F(y) \text { for } x, y \in[0, \infty),
$$

Communicated by A. Constantin.

$凶 \quad$ J. Chudziak

chudziak@univ.rzeszow.pl; chudziak@ur.edu.pl

Z. Kočan

zdenek.kocan@math.slu.cz

1 Faculty of Mathematics and Natural Sciences, University of Rzeszów, St. Pigonia 1, 35-959

Rzeszow, Poland

2 Mathematical Institute in Opava, Silesian University in Opava, Na Rybníčku 626/1, 746 01 Opava, Czech Republic 
where $r$ is a positive real constant. It turns out that Eq. (1) by some simple substitutions can be reduced to the equation

$$
f(x+f(x) y)=f(x) f(y) \text { for } x, y \in[0, \infty),
$$

which is the Gołąb-Schinzel equation on a restricted domain. More details on the Gołąb-Schinzel type functional equation can be found in [3]. Continuous solutions $f: \mathbb{R} \rightarrow \mathbb{R}$ of Eq. (2) have been determined in [1]. Applying the results of [1], P. Kahlig and J. Matkowski [15] have determined the solutions of (1) in the class of pairs of functions $(F, s)$, where $F:[0, \infty) \rightarrow[0, \infty)$ is continuous (or monotone) and $s:[0, \infty) \rightarrow \mathbb{R}$. Inspired by this fact several authors have considered conditional versions of the Gołąb-Schinzel equation. In particular the solutions of the equation

$$
f(x+f(x) y)=f(x) f(y) \text { whenever } x, y, x+f(x) y \geq 0
$$

has been considered in [19]. For further results concerning conditional Gołąb-Schinzel type functional equations we refer to [2], [4], [17]-[18] and [20]-[21].

In a series of papers [7], [9] and [12]-[14] the following generalization of the Gołąb-Schinzel equation

$$
F(x+G(x) y)=F(x) F(y)
$$

has been studied in various settings. Further going pexiderization of the Gołąb-Schinzel equation have been investigated in [5]-[6] and [10]-[11].

In a recent paper [8] the results of [1] and [19] have been generalized. More precisely, the continuous solutions of the equations

$$
f(x+g(x) y)=f(x) f(y) \text { whenever } \quad x, y, x+g(x) y \geq 0
$$

and

$$
f(x+g(x) y)=f(x) f(y) \text { for } x, y \in[0, \infty)
$$

have been determined.

In the present paper, applying the results of [8], we deal with a similar problem, but in a much more general setting. Namely, given a real linear space $X$ and a convex cone $\mathcal{C}$ in $X$, that is a nonempty subset of $X$ such that $\alpha x+\beta y \in \mathcal{C}$ for $x, y \in \mathcal{C}$ and $\alpha, \beta \in[0, \infty)$, we determine the solutions of the equation

$$
f(x+g(x) y)=f(x) f(y) \quad \text { whenever } \quad x, y, x+g(x) y \in \mathcal{C}
$$

in the class of pairs $(f, g)$ of functions continuous on rays mapping $\mathcal{C}$ into $\mathbb{R}$. Roughly speaking, our main result says that the pair $(f, g)$ of functions continuous on rays satisfies Eq. (6) if and only if there exist continuous functions $\tilde{f}, \tilde{g}: \mathbb{R} \rightarrow \mathbb{R}$ and a nontrivial linear functional $L: \operatorname{Lin} \mathcal{C} \rightarrow \mathbb{R}$ such that the pair $(\tilde{f}, \tilde{g})$ satisfies Eq. (4), $f=\tilde{f} \circ L_{\mid \mathcal{C}}$ and either $g=\tilde{g} \circ L_{\mid \mathcal{C}}$, or $L(\mathcal{C})=(-\infty, 0]$ and, for every $x \in \mathcal{C}$, 
$g(x)=(\tilde{g} \circ L)(x)$ whenever $1+L(x)>0$ and $g(x) \geq 0$, otherwise. Furthermore we show that the similar assertion holds for the solution of the equation

$$
F(x+G(x) y)=F(x) F(y) \text { for } x, y \in \mathcal{C}
$$

in the class of pairs $(F, G)$ of functions continuous on rays mapping $X$ into $\mathbb{R}$. Let us recall that a function $F: X \rightarrow \mathbb{R}$ is said to be continuous on rays provided, for every $x \in X$, the function $F_{x}: \mathbb{R} \rightarrow \mathbb{R}$ given by $F_{x}(t)=F(t x)$ for $t \in \mathbb{R}$, is continuous. A function $f: \mathcal{C} \rightarrow \mathbb{R}$ is said to be continuous on rays provided, for every $x \in \mathcal{C}$, the function $f_{x}:[0, \infty) \rightarrow \mathbb{R}$ given by $f_{x}(t)=f(t x)$ for $t \in[0, \infty)$, is continuous.

In what follows $X$ stands for a real linear space and $\mathcal{C}$ denotes a convex cone in $X$. These assumptions will not be repeated.

\section{Preliminary results}

The following result will play a crucial role in our considerations [cf. [8, Theorem 3.1]]. So, we provide it here for reader's convenience.

Theorem 2.1 Assume that $f, g:[0, \infty) \rightarrow \mathbb{R}$ are continuous functions. Then the pair $(f, g)$ satisfies Eq. (4) if and only if one of the subsequent possibilities holds:

(i) $f(x)=0$ for $x \in[0, \infty)$ and $g$ is arbitrary;

(ii) $f(x)=1$ for $x \in[0, \infty)$ and $g$ is arbitrary;

(iii) $g(x)=1$ for $x \in[0, \infty)$ and there exists a $c \in \mathbb{R} \backslash\{0\}$ such that

$$
f(x)=e^{c x} \text { for } x \in[0, \infty)
$$

(iv) There exist a $c \in(0, \infty)$ and an $r \in \mathbb{R} \backslash\{0\}$ such that

$$
g(x)=1+c x \quad \text { for } \quad x \in[0, \infty)
$$

and

$$
f(x)=(1+c x)^{r} \quad \text { for } \quad x \in[0, \infty)
$$

(v) There exist a $c \in(-\infty, 0)$ and an $r \in(0, \infty)$ such that $g$ is of the form (8) and either

$$
f(x)=|1+c x|^{r} \quad \text { for } \quad x \in[0, \infty)
$$

or

$$
f(x)=|1+c x|^{r} \cdot \operatorname{sgn}(1+c x) \text { for } x \in[0, \infty) ;
$$

(vi) There exist a $c \in(-\infty, 0)$, an $r \in(0, \infty)$ and a continuous function $\phi$ : $\left[-\frac{1}{c}, \infty\right) \rightarrow[0, \infty)$ such that $\phi\left(-\frac{1}{c}\right)=0$,

$$
g(x)= \begin{cases}1+c x & \text { for } x \in\left[0,-\frac{1}{c}\right) \\ \phi(x) & \text { for } x \in\left[-\frac{1}{c}, \infty\right)\end{cases}
$$


and

$$
f(x)=(\max \{1+c x, 0\})^{r} \text { for } x \in[0, \infty) .
$$

Lemma 2.1 Assume that $f, g: \mathcal{C} \rightarrow \mathbb{R}$ and the pair $(f, g)$ satisfies Eq. (6). Then either $f=0$ or $f(0)=1$.

Proof Applying (6) with $x=y=0$, we get $f(0) \in\{0,1\}$. Furthermore, if $f(0)=0$ then putting in (6) $y=0$ we obtain that $f=0$.

Lemma 2.2 Assume that functions $f, g: \mathcal{C} \rightarrow \mathbb{R}$ are continuous on rays, $f \neq 0$ and the pair $(f, g)$ satisfies $E q$. (6). Then, for every $x \in \mathcal{C}$, one of the following possibilities holds:

$\left(C_{1}\right) f_{x}(t)=1$ for $t \in[0, \infty)$;

$\left(C_{2}\right) g_{x}(t)=1$ for $t \in[0, \infty)$ and there exists a $c(x) \in \mathbb{R} \backslash\{0\}$ such that

$$
f_{x}(t)=e^{c(x) t} \text { for } t \in[0, \infty)
$$

$\left(C_{3}\right)$ there exist a $c(x) \in(0, \infty)$ and an $r(x) \in \mathbb{R} \backslash\{0\}$ such that

$$
g_{x}(t)=1+c(x) t \quad \text { for } t \in[0, \infty)
$$

and

$$
f_{x}(t)=(1+c(x) t)^{r(x)} \text { for } t \in[0, \infty) ;
$$

$\left(C_{4}\right)$ there exist a $c(x) \in(-\infty, 0)$ and an $r(x) \in(0, \infty)$ such that $g_{x}$ is of the form (11) and

$$
f_{x}(t)=|1+c(x) t|^{r(x)} \text { for } t \in[0, \infty)
$$

$\left(C_{5}\right)$ there exist a $c(x) \in(-\infty, 0)$ and an $r(x) \in(0, \infty)$ such that $g_{x}$ is of the form (11) and

$$
f_{x}(t)=|1+c(x) t|^{r(x)} \cdot \operatorname{sgn}(1+c(x) t) \text { for } t \in[0, \infty)
$$

$\left(C_{6}\right)$ there exist a $c(x) \in(-\infty, 0)$, an $r(x) \in(0, \infty)$ and a continuous function $\phi^{(x)}:\left[-\frac{1}{c(x)}, \infty\right) \rightarrow[0, \infty)$ such that $\phi^{(x)}\left(-\frac{1}{c(x)}\right)=0$,

$$
g_{x}(t)= \begin{cases}1+c(x) t & \text { for } t \in\left[0,-\frac{1}{c(x)}\right), \\ \phi^{(x)}(t) & \text { for } t \in\left[-\frac{1}{c(x)}, \infty\right)\end{cases}
$$

and

$$
f_{x}(t)=(\max \{1+c(x) t, 0\})^{r(x)} \text { for } t \in[0, \infty) .
$$

Proof According to Lemma 2.1, we get $f(0)=1$. Moreover, in view of (6), for every $x \in \mathcal{C}$ and $s, t \in[0, \infty)$ such that $s x+g(s x) t x \in \mathcal{C}$, we obtain 


$$
f_{x}\left(s+g_{x}(s) t\right)=f(s x+g(s x) t x)=f(s x) f(t x)=f_{x}(s) f_{x}(t) .
$$

Furthermore, for every $x \in \mathcal{C}$ and $s, t \in[0, \infty)$, we have $s x+g(s x) t x=\left(s+g_{x}(s) t\right) x$, that is $s x+g(s x) t x \in \mathcal{C}$ provided $s+g_{x}(s) t \geq 0$. Therefore, for every $x \in \mathcal{C}$, the pair $\left(f_{x}, g_{x}\right)$ satisfies Eq. (4). Since, for every $x \in \mathcal{C}, f_{x}$ and $g_{x}$ are continuous and $f_{x}(0)=f(0)=1$, applying Theorem 2.1, we get the assertion.

Remark 2.1 In the sequel, for every $i \in\{1,2,3,4,5,6\}$, we set

$$
\mathcal{C}_{(i)}:=\left\{x \in \mathcal{C} \mid\left(C_{i}\right) \text { holds for } x\right\}
$$

According to Lemma 2.2, if $f \neq 0$ then $0 \in \mathcal{C}_{(1)}, \bigcup_{i=1}^{6} \mathcal{C}_{(i)}=\mathcal{C}$ and

$$
\mathcal{C}_{(i)} \cap \mathcal{C}_{(j)}=\varnothing \text { for } i, j \in\{1,2,3,4,5,6\}, i \neq j
$$

Furthermore, for every $i \in\{1,2,3,4,5,6\}$, we have

$$
\lambda x \in \mathcal{C}_{(i)} \text { whenever } x \in \mathcal{C}_{(i)} \text { and } \lambda \in(0, \infty) .
$$

Note also that $g_{x}(0)=1$ for $x \in \bigcup_{i=2}^{6} \mathcal{C}_{(i)}$, so $g(0)=1$ provided $\bigcup_{i=2}^{6} \mathcal{C}_{(i)} \neq \emptyset$.

Lemma 2.3 Assume that functions $f, g: \mathcal{C} \rightarrow \mathbb{R}$ are continuous on rays, $f$ is nonconstant and the pair ( $f, g$ ) satisfies Eq. (6). Then

$$
g_{x}(t)>0 \text { for } x \in \mathcal{C}_{(1)}, t \in[0, \infty) .
$$

Proof Since $f$ is nonconstant, according to Remark 2.1, we have $\bigcup_{i=2}^{6} \mathcal{C}_{(i)} \neq \emptyset$ and so $g(0)=1$. Suppose that $g_{x}(t) \leq 0$ for some $x \in \mathcal{C}_{(1)}$ and $t \in[0, \infty)$. As $g_{x}$ is continuous and $g_{x}(0)=g(0)=1$, this means that $t>0$ and $g\left(t_{0} x\right)=g_{x}\left(t_{0}\right)=0$ for some $t_{0} \in(0, t]$. Hence $t_{0} x+g\left(t_{0} x\right) y=t_{0} x \in \mathcal{C}$ for $y \in \mathcal{C}$ and so, in view of (6), we obtain

$$
1=f_{x}\left(t_{0}\right)=f\left(t_{0} x\right)=f\left(t_{0} x+g\left(t_{0} x\right) y\right)=f\left(t_{0} x\right) f(y)=f_{x}\left(t_{0}\right) f(y)=f(y)
$$

for $y \in \mathcal{C}$, which yields a contradiction.

In the proof of the next proposition we will need the following lemma.

Lemma 2.4 Assume that $n \in \mathbb{N}, \gamma_{i} \in \mathbb{R}$ for $i \in\{0, \ldots, n\}, \beta_{i} \in \mathbb{R} \backslash\{0\}$ for $i \in$ $\{1, \ldots, n\}$ and $\beta_{j} \neq \beta_{k}$ for $j, k \in\{1, \ldots, n\}, j \neq k$. Suppose that

$$
\sum_{j=1}^{n} \gamma_{j} \ln \left(1+\beta_{j} t\right)=\gamma_{0} t \quad \text { for } \quad t \in[0, \varepsilon)
$$

where $\varepsilon \in(0, \infty)$ is fixed. Then $\gamma_{i}=0$ for $i \in\{0, \ldots, n\}$. 
Proof Differentiating (20) with respect to $t$, for every $k \in\{2, \ldots, n+1\}$, we obtain

$$
\sum_{j=1}^{n} \frac{\beta_{j}^{k} \gamma_{j}}{\left(1+\beta_{j} t\right)^{k}}=0 \text { for } t \in(0, \varepsilon) .
$$

Thus, letting $t \rightarrow 0^{+}$and setting $\delta_{j}:=\beta_{j}^{2} \gamma_{j}$ for $j \in\{1, \ldots, n\}$, we get

$$
\sum_{j=1}^{n} \beta_{j}^{k} \delta_{j}=0 \text { for } k \in\{0, \ldots, n-1\} .
$$

The determinant of this system of $n$ linear equations with variables $\delta_{1}, \ldots, \delta_{n}$ is the Vandermonde determinant. Hence, as $\beta_{j} \neq \beta_{k}$ for $j, k \in\{1, \ldots, n\}, j \neq k$, it is different from 0 . Thus $\delta_{i}=0$ for $i \in\{1, \ldots, n\}$. Since $\beta_{i} \neq 0$ for $i \in\{1, \ldots, n\}$, this means that $\gamma_{i}=0$ for $i \in\{1, \ldots, n\}$ which, in view of (20), gives $\gamma_{0}=0$.

Proposition 2.1 Assume that functions $f, g: \mathcal{C} \rightarrow \mathbb{R}$ are continuous on rays and the pair $(f, g)$ satisfies Eq. (6). If $\mathcal{C}_{(2)} \neq \emptyset$ then $g=1$ and there exists a nontrivial linear functional $L:$ Lin $\mathcal{C} \rightarrow \mathbb{R}$ such that $f(x)=e^{L(x)}$ for $x \in \mathcal{C}$.

Proof Assume that $\mathcal{C}_{(2)} \neq \varnothing$. First we show that $\mathcal{C}=\mathcal{C}_{(1)} \cup \mathcal{C}_{(2)}$. Suppose that this is not true. Since $\mathcal{C}_{(2)} \neq \varnothing$, we have $f \neq 0$ and so, according to Remark 2.1, we get $\bigcup_{i=3}^{6} \mathcal{C}_{(i)} \neq \varnothing$. Let $y \in \bigcup_{i=3}^{6} \mathcal{C}_{(i)}$. Then there exist $c(y), r(y) \in \mathbb{R} \backslash\{0\}$ such that

$$
f_{y}(t)=(1+c(y) t)^{r(y)} \text { for sufficiently small } t \geq 0 .
$$

Fix an $x \in \mathcal{C}_{(2)}$. Then $t x+g(t x) t y=t x+g_{x}(t) t y=t x+t y \in \mathcal{C}$ for $t \in[0, \infty)$ and so, taking into account (6) and (21), we get

$$
\begin{aligned}
f_{x+y}(t) & =f(t x+t y)=f(t x+g(t x) t y)=f(t x) f(t y) \\
& =f_{x}(t) f_{y}(t)=e^{c(x) t}(1+c(y) t)^{r(y)} \quad \text { for sufficiently small } \quad t \geq 0 .
\end{aligned}
$$

If $x+y \in \mathcal{C}_{(1)} \cup \mathcal{C}_{(2)}$ then $f_{x+y}(t)=e^{c(x+y) t}$ for $t \in[0, \infty)$ with some $c(x+y) \in \mathbb{R}$ and so from (22) we derive that

$$
r(y) \ln (1+c(y) t)=(c(x+y)-c(x)) t \text { for sufficiently small } t \geq 0 .
$$

Thus, as $c(y) \neq 0$, according to Lemma 2.4, we get $r(y)=0$, which yields a contradiction. If $x+y \in \bigcup_{i=3}^{6} \mathcal{C}_{(i)}$ then there exist $c(x+y), r(x+y) \in \mathbb{R} \backslash\{0\}$ such that $f_{x+y}(t)=(1+c(x+y) t)^{r(x+y)}$ for sufficiently small $t \geq 0$. Hence, in view of (22), we get

$$
\begin{aligned}
& r(x+y) \ln (1+c(x+y) t)-r(y) \ln (1+c(y) t) \\
& \quad=c(x) t \quad \text { for sufficiently small } t \geq 0 .
\end{aligned}
$$


Therefore, as $c(x+y) \neq 0$ and $c(y) \neq 0$, in the case where $c(x+y) \neq c(y)$, applying Lemma 2.4, we conclude that $r(x+y)=r(y)=c(x)=0$, which yields a contradiction. If $c(x+y)=c(y)$ then we have

$$
(r(x+y)-r(y)) \ln (1+c(y) t)=c(x) t \text { for sufficiently small } t \geq 0 .
$$

Thus, as $c(y) \neq 0$, according to Lemma 2.4, we obtain that $c(x)=0$ which again gives a contradiction.

In this way we have proved that $\mathcal{C}=\mathcal{C}_{(1)} \cup \mathcal{C}_{(2)}$. Therefore, according to Lemma 2.3, we get $g_{x}(t)>0$ for $x \in \mathcal{C}, t \in[0, \infty)$ and so from (6) we derive that

$$
f(x+g(x) y)=f(x) f(y) \text { for } x, y \in \mathcal{C} .
$$

Moreover, for every $x \in \mathcal{C}$, there exists a $c(x) \in \mathbb{R}$ such that

$$
f_{x}(t)=e^{c(x) t} \quad \text { for } \quad t \in[0, \infty)
$$

In view of (24), for every $x \in \mathcal{C}$ and $\alpha, t \in[0, \infty)$, we get

$$
e^{c(\alpha x) t}=f_{\alpha x}(t)=f_{x}(\alpha t)=e^{c(x) \alpha t},
$$

which implies that

$$
c(\alpha x)=\alpha c(x) \text { for } x \in \mathcal{C}, \alpha \in[0, \infty)
$$

We claim that

$$
c(x+y)=c(x)+c(y) \text { for } x, y \in \mathcal{C} .
$$

Fix $x, y \in \mathcal{C}$. If $x \in \mathcal{C}_{(1)}$ then $c(x)=0$ whence, in view of (23) and (24), we obtain

$$
\begin{aligned}
e^{c(x+y) t} & =f_{x+y}(t)=f(t x+t y)=f\left(t y+g(t y) \frac{t x}{g(t y)}\right) \\
& =f(t y) f\left(\frac{t x}{g(t y)}\right)=f_{y}(t) f_{x}\left(\frac{t}{g(t y)}\right)=f_{y}(t)=e^{c(y) t} \text { for } t \in[0, \infty)
\end{aligned}
$$

Hence $c(x+y)=c(y)=c(x)+c(y)$. If $x \in \mathcal{C}_{(2)}$ then from (23) it follows that $f(x+y)=f(x) f(y)$. Since, in view of (24), $f(x+y)=f_{x+y}(1)=e^{c(x+y)}, f(x)=$ $f_{x}(1)=e^{c(x)}$ and $f(y)=f_{y}(1)=e^{c(y)}$, this implies that $c(x+y)=c(x)+c(y)$. Thus (26) is proved.

Now, applying [16, Theorem 4.4.1, p. 88], from (25) and (26) we derive that there exists a linear functional $L: \operatorname{Lin} \mathcal{C} \rightarrow \mathbb{R}$ such that

$$
c(x)=L(x) \text { for } x \in \mathcal{C} .
$$

Hence, according to (24), $f(x)=f_{x}(1)=e^{L(x)} \quad$ for $\quad x \in \mathcal{C}$. Furthermore, as $\mathcal{C}_{(2)} \neq \varnothing$, we get that $L_{\mid \mathcal{C}}$ is not identically zero and so $L$ is nontrivial. Finally, 
inserting into (23) $f$ of the above form, we obtain $(g(x)-1) L(y)=0$ for $x, y \in \mathcal{C}$. Since $\left.L\right|_{\mathcal{C}}$ is not identically zero, this means that $g=1$.

Proposition 2.2 Assume that functions $f, g: \mathcal{C} \rightarrow \mathbb{R}$ are continuous on rays, $f$ is nonconstant and the pair $(f, g)$ satisfies Eq. (6). If $\mathcal{C}_{(2)}=\emptyset$ then $\bigcup_{i=3}^{6} \mathcal{C}_{(i)} \neq \varnothing$ and there exist an $r \in \mathbb{R} \backslash\{0\}$ and a nontrivial linear functional $L:$ Lin $\mathcal{C} \rightarrow \mathbb{R}$ such that $r>0$ whenever $\bigcup_{i=4}^{6} \mathcal{C}_{(i)} \neq \emptyset, \mathcal{C}_{(1)}=\mathcal{C} \cap \operatorname{ker} L$ and:

$(\alpha)$ If $x \in \mathcal{C}_{(3)}$ then $L(x)>0, f(x)=(1+L(x))^{r}$ and

$$
g(x)=1+L(x)
$$

( $\beta)$ If $x \in \mathcal{C}_{(4)}$ then $L(x)<0$, (28) holds and $f(x)=|1+L(x)|^{r}$;

$(\gamma)$ If $x \in \mathcal{C}_{(5)}$ then $L(x)<0$, (28) holds and $f(x)=|1+L(x)|^{r} \operatorname{sgn}(1+L(x))$;

( $\delta)$ If $x \in \mathcal{C}_{(6)}$ then $L(x)<0$,

$$
\begin{gathered}
f(x)=(\max \{1+L(x), 0\})^{r}, \\
g(x)=1+L(x) \text { whenever } 1+L(x)>0,
\end{gathered}
$$

and

$$
g(x) \geq 0 \text { whenever } 1+L(x) \leq 0 .
$$

Proof Assume that $\mathcal{C}_{(2)}=\emptyset$. Since $f$ is nonconstant, we have $\mathcal{C}_{(1)} \neq \mathcal{C}$ and so, according to Remark 2.1, we get $\bigcup_{i=3}^{6} \mathcal{C}_{(i)} \neq \emptyset$. Let

$$
V_{x}= \begin{cases}{[0, \infty)} & \text { for } x \in \mathcal{C}_{(1)} \cup \mathcal{C}_{(3)} \\ {\left[0,-\frac{1}{c(x)}\right)} & \text { for } x \in \mathcal{C}_{(4)} \cup \mathcal{C}_{(5)} \cup \mathcal{C}_{(6)}\end{cases}
$$

Then, taking into account (11) and (15), in view of Lemma 2.3, we obtain

$$
g(t x)=g_{x}(t)>0 \text { for } x \in \mathcal{C}, t \in V_{x}
$$

Thus, for every $x, y \in \mathcal{C}$ and $t \in V_{x}$, we have $\frac{t}{g(t x)} y \in \mathcal{C}$ and $t x+g(t x) \frac{t}{g(t x)} y=$ $t x+t y \in \mathcal{C}$. Therefore, making use of (6), for every $x, y \in \mathcal{C}$ and $t \in V_{x}$, we obtain

$$
f(t x+t y)=f\left(t x+g(t x) \frac{t}{g(t x)} y\right)=f(t x) f\left(\frac{t}{g(t x)} y\right) .
$$

Hence

$$
f_{x+y}(t)=f_{x}(t) f_{y}\left(\frac{t}{g_{x}(t)}\right) \text { for } x, y \in \mathcal{C}, t \in V_{x} .
$$

In the same way, we get

$$
f_{y+x}(t)=f_{y}(t) f_{x}\left(\frac{t}{g_{y}(t)}\right) \text { for } x, y \in \mathcal{C}, t \in V_{y} .
$$


Moreover, from (12)-(14) and (16) it follows that

$$
f_{x}(t)=(1+c(x) t)^{r(x)} \text { for } x \in \bigcup_{i=3}^{6} \mathcal{C}_{(i)}, t \in V_{x}
$$

Note also that, for every $x, y \in \bigcup_{i=3}^{6} \mathcal{C}_{(i)}$ and sufficiently small $t \in V_{x} \cap V_{y}$, we have $\frac{t}{g_{x}(t)}=\frac{t}{1+c(x) t} \in V_{y}$ and $\frac{t}{g_{y}(t)}=\frac{t}{1+c(y) t} \in V_{x}$. Therefore, taking into account (33)-(35), we conclude that

$$
(1+c(x) t)^{r(x)}\left(1+\frac{c(y) t}{1+c(x) t}\right)^{r(y)}=(1+c(y) t)^{r(y)}\left(1+\frac{c(x) t}{1+c(y) t}\right)^{r(x)}
$$

for every $x, y \in \bigcup_{i=3}^{6} \mathcal{C}_{(i)}$ and sufficiently small $t \in V_{x} \cap V_{y}$. Hence

$$
\left(1+\frac{c(x) c(y) t^{2}}{1+(c(x)+c(y)) t}\right)^{r(x)-r(y)}=1
$$

for every $x, y \in \bigcup_{i=3}^{6} \mathcal{C}_{(i)}$ and sufficiently small $t \in V_{x} \cap V_{y}$. Since $c(x) c(y) \neq 0$ for $x, y \in \bigcup_{i=3}^{6} \mathcal{C}_{(i)}$, this implies that $r(x)=r(y)$ for $x, y \in \bigcup_{i=3}^{6} \mathcal{C}_{(i)}$. Thus there exists an $r \in \mathbb{R} \backslash\{0\}$ such that

$$
r(x)=r \text { for } x \in \bigcup_{i=3}^{6} \mathcal{C}_{(i)}
$$

Moreover, according to Lemma 2.2, we get $r>0$ whenever $\bigcup_{i=4}^{6} \mathcal{C}_{(i)} \neq \emptyset$. Furthermore, taking

$$
c(x)=0 \text { for } x \in \mathcal{C}_{(1)},
$$

in view of (35), we obtain

$$
f_{x}(t)=(1+c(x) t)^{r} \text { for } \quad x \in \mathcal{C}, t \in V_{x} .
$$

Thus, for every $x \in \mathcal{C}$ and $\alpha \in(0, \infty)$, we get

$$
(1+c(\alpha x) t)^{r}=f_{\alpha x}(t)=f_{x}(\alpha t)=(1+c(x) \alpha t)^{r} \text { for } t \in \frac{1}{\alpha} V_{x} \cap V_{\alpha x},
$$

which implies (25). Next we show that (26) is valid. Fix $x, y \in \mathcal{C}$. If $y \in \mathcal{C}_{(1)}$ then, taking into account (33), we get $f_{x+y}(t)=f_{x}(t)$ for $t \in V_{x}$. Therefore, in view of (37), we obtain $(1+c(x+y) t)^{r}=(1+c(x) t)^{r}$ for $t \in V_{x} \cap V_{x+y}$. Hence $c(x+y)=c(x)$ and so, according to (36), we get $c(x+y)=c(x)+c(y)$. In the case where $x \in \mathcal{C}_{(1)}$, taking into account (34), we obtain the same assertion. Assume that $x, y \in \bigcup_{i=3}^{6} \mathcal{C}_{(i)}$. 
Since, as we have already noted, $\frac{t}{g_{x}(t)}=\frac{t}{1+c(x) t} \in V_{y}$ for sufficiently small $t \in V_{x}$, making use of (33) and (37), we obtain

$$
(1+c(x+y) t)^{r}=(1+c(x) t)^{r}\left(1+\frac{c(y) t}{1+c(x) t}\right)^{r}=(1+(c(x)+c(y)) t)^{r}
$$

for sufficiently small $t \in V_{x} \cap V_{x+y}$. Thus $c(x+y)=c(x)+c(y)$ and so (26) is proved. Therefore, applying [16, Theorem 4.4.1, p. 88], from (25) and (26) we deduce that there exists a linear functional $L: \operatorname{Lin} \mathcal{C} \rightarrow \mathbb{R}$ such that (27) holds. Since $\bigcup_{i=3}^{6} \mathcal{C}_{(i)} \neq \emptyset, L$ is nontrivial. Furthermore, for every $x \in \mathcal{C}$, we have $f(x)=f_{x}(1)$ and $g(x)=g_{x}(1)$, so taking into account (27) and applying Lemma 2.2, we obtain $(\alpha)-(\delta)$. From $(\alpha)-(\delta)$ it follows that $\mathcal{C} \cap \operatorname{ker} L \subseteq \mathcal{C}_{(1)}$. On the other hand, (27) and (36) imply that $\mathcal{C}_{(1)} \subseteq \mathcal{C} \cap \operatorname{ker} L$. Thus $\mathcal{C}_{(1)}=\mathcal{C} \cap \operatorname{ker} L$.

\section{Main results}

The next theorem is the main result of the paper.

Theorem 3.1 Assume that functions $f, g: \mathcal{C} \rightarrow \mathbb{R}$ are continuous on rays. Then the pair $(f, g)$ satisfies Eq. (6) if and only if one of the following possibilities holds:

(a) $f=0$ and $g$ is arbitrary;

(b) $f=1$ and $g$ is arbitrary;

(c) $g=1$ and there exists a nontrivial linear functional $L:$ Lin $\mathcal{C} \rightarrow \mathbb{R}$ such that $f(x)=e^{L(x)}$ for $x \in \mathcal{C}$;

(d) There exist an $r \in \mathbb{R} \backslash\{0\}$ and a nontrivial linear functional $L:$ Lin $\mathcal{C} \rightarrow \mathbb{R}$ such that $r>0$ whenever $L(\mathcal{C}) \cap(-\infty, 0) \neq \emptyset$,

$$
g(x)=\max \{L(x)+1,0\} \text { for } x \in \mathcal{C}
$$

and

$$
f(x)=(\max \{1+L(x), 0\})^{r} \quad \text { for } \quad x \in \mathcal{C} ;
$$

(e) There exist an $r \in(0, \infty)$ and a linear functional $L:$ Lin $\mathcal{C} \rightarrow \mathbb{R}$ such that $L(\mathcal{C}) \cap(-\infty, 0) \neq \emptyset$,

$$
g(x)=1+L(x) \text { for } x \in \mathcal{C},
$$

and either

$$
f(x)=|1+L(x)|^{r} \quad \text { for } \quad x \in \mathcal{C}
$$

or

$$
f(x)=|1+L(x)|^{r} \operatorname{sgn}(1+L(x)) \text { for } x \in \mathcal{C} ;
$$

(f) There exist an $r \in(0, \infty)$ and a linear functional $L:$ Lin $\mathcal{C} \rightarrow \mathbb{R}$ such that $L(\mathcal{C})=(-\infty, 0], f$ is of the form (39) and, for every $x \in \mathcal{C},(30)-(31)$ hold. 
Proof A straightforward calculation shows that if one of the possibilities $(a)-(f)$ holds then the pair $(f, g)$ satisfies Eq. (6).

Assume that the pair $(f, g)$ satisfies Eq. (6). If $f$ is constant then, in view of Lemma 2.1, $f=0$ or $f=1$ and so, either $(a)$ or $(b)$ holds. Furthermore, if $\mathcal{C}_{(2)} \neq \varnothing$ then, applying Proposition 2.1, we get $(c)$. So, assume that $f$ is nonconstant and $\mathcal{C}_{(2)}=\emptyset$. Then, according to Proposition 2.2, $\bigcup_{i=3}^{6} \mathcal{C}_{(i)} \neq \emptyset$ and there exist an $r \in \mathbb{R} \backslash\{0\}$ and a nontrivial linear functional $L: \operatorname{Lin} \mathcal{C} \rightarrow \mathbb{R}$ such that $(\alpha)-(\delta)$ hold, $r>0$ whenever $\bigcup_{i=4}^{6} \mathcal{C}_{(i)} \neq \emptyset$ and $\mathcal{C}_{(1)}=\mathcal{C} \cap \operatorname{ker} L$. In particular, for every $x \in \mathcal{C}$, we obtain

$$
f(x)=(1+L(x))^{r} \text { whenever } 1+L(x)>0 .
$$

Note also that, according to Lemma 2.3, for every $x \in \mathcal{C}_{(1)}$ and $y \in \mathcal{C}$, we get $x+g(x) t y \in \mathcal{C}$ for $t \in[0, \infty)$. Moreover, we have

$$
1+L(x+g(x) t y)=1+g(x) t L(y)>0 \text { for sufficiently small } t \in[0, \infty) .
$$

Thus, in view of (43), we obtain

$$
f(x+g(x) t y)=(1+g(x) t L(y))^{r} \text { for sufficiently small } t \in[0, \infty) .
$$

On the other hand, making use of (6) and (43), for every $x \in \mathcal{C}_{(1)}$ and $y \in \mathcal{C}$, we get

$$
\begin{aligned}
& f(x+g(x) t y)=f(x) f(t y)=f(t y) \\
& \quad=(1+t L(y))^{r} \text { for sufficiently small } t \in[0, \infty) .
\end{aligned}
$$

Hence

$$
g(x)=1 \text { for } x \in \mathcal{C}_{(1)} .
$$

Therefore, taking into account $(\alpha)-(\delta)$, we conclude that:

- If $\mathcal{C}_{(4)}=\mathcal{C}_{(5)}=\mathcal{C}_{(6)}=\emptyset$ then $(d)$ holds;

- If $\mathcal{C}_{(4)} \neq \emptyset$ and $\mathcal{C}_{(5)}=\mathcal{C}_{(6)}=\emptyset$, or $\mathcal{C}_{(5)} \neq \emptyset$ and $\mathcal{C}_{(4)}=\mathcal{C}_{(6)}=\varnothing$ then $(e)$ holds;

- If $\mathcal{C}_{(3)}=\mathcal{C}_{(4)}=\mathcal{C}_{(5)}=\emptyset$ and $\mathcal{C}_{(6)} \neq \emptyset$ then $(f)$ holds.

Suppose that $\mathcal{C}_{(3)} \neq \emptyset, \mathcal{C}_{(6)} \neq \emptyset$ and $\mathcal{C}_{(4)}=\mathcal{C}_{(5)}=\emptyset$. We claim that in this case $(d)$ is valid. In view of (29)-(30), in order to show this, it is enough to prove that $g(x)=0$ for every $x \in \mathcal{C}$ with $1+L(x) \leq 0$. Fix an $x \in \mathcal{C}$ such that $1+L(x) \leq 0$. Then $x \in \mathcal{C}_{(6)}$ and so, in view of (31), we obtain that $g(x) \geq 0$. Thus, taking a $y \in \mathcal{C}_{(3)}$, we get $x+g(x) s y \in \mathcal{C}$ for $s \in[0, \infty)$. Hence, making use of (6) and (29), we obtain

$$
f(x+g(x) s y)=f(x) f(s y)=0 \text { for } s \in[0, \infty) .
$$

On the other hand, according to Proposition 2.2, we have $f(z) \geq 1$ for $z \in \mathcal{C}_{(1)} \cup \mathcal{C}_{(3)}$. Thus from (45) we derive that $x+g(x) s y \notin \mathcal{C}_{(1)} \cup \mathcal{C}_{(3)}$ for $s \in[0, \infty)$ and so, as $\mathcal{C}_{(4)}=\mathcal{C}_{(5)}=\emptyset$, we get $x+g(x) s y \in \mathcal{C}_{(6)}$ for $s \in[0, \infty)$. Therefore, taking into account (29) and (45), we obtain

$$
1+L(x)+g(x) s L(y)=1+L(x+g(x) s y) \leq 0 \text { for } s \in[0, \infty) .
$$


Furthermore, $L(y)>0$ because $y \in \mathcal{C}_{(3)}$. Hence, we have $g(x)=0$.

Now, in order to complete the proof, it is enough to show that at most one of the sets $\mathcal{C}_{(i)}$ for $i \in\{4,5,6\}$ is nonempty. For the proof by contradiction suppose that $\mathcal{C}_{\left(j_{1}\right)} \neq \emptyset$ and $\mathcal{C}_{\left(j_{2}\right)} \neq \emptyset$ for some $j_{1}, j_{2} \in\{4,5,6\}, j_{1} \neq j_{2}$. Fix an $x \in \mathcal{C}_{\left(j_{1}\right)}$, a $y \in \mathcal{C}_{\left(j_{2}\right)}$ and put $\mathcal{C}(x, y):=\{\alpha x+\beta y: \alpha, \beta \in(0, \infty)\}$ and $\overline{\mathcal{C}}(x, y):=\{\alpha x+\beta y: \alpha, \beta \in$ $[0, \infty), \alpha+\beta>0\}$. Obviously $\emptyset \neq \mathcal{C}(x, y) \subset \overline{\mathcal{C}}(x, y)$. Moreover, as $L(x), L(y)<0$, we have $L(z)<0$ for $z \in \overline{\mathcal{C}}(x, y)$, that is

$$
\overline{\mathcal{C}}(x, y) \subseteq \mathcal{C}_{(4)} \cup \mathcal{C}_{(5)} \cup \mathcal{C}_{(6)}
$$

Thus one of the following two cases is possible:

1. $\mathcal{C}(x, y) \cap \mathcal{C}_{(k)} \neq \emptyset$ for some $k \in\{4,5\}$,

2. $\mathcal{C}(x, y) \subseteq \mathcal{C}_{(6)}$.

Case 1 Let $z \in \mathcal{C}(x, y) \cap \mathcal{C}_{(k)}$. Then $L(z)<0$ and so $\lambda_{n}:=-\left(1+\frac{1}{n}\right) \frac{1}{L(z)}>0$ for $n \in \mathbb{N}$. Put $z_{n}:=\lambda_{n} z$ for $n \in \mathbb{N}$. According to (18), $z_{n} \in \mathcal{C}_{(k)}$ for $n \in \mathbb{N}$, which in view of (28), gives

$$
g\left(z_{n}\right)=1+L\left(z_{n}\right)=1+\lambda_{n} L(z)=-\frac{1}{n} \text { for } n \in \mathbb{N} .
$$

Furthermore, for every $n \in \mathbb{N}$, we have $z_{n} \in \mathcal{C}(x, y)$, so there exist $\alpha_{n}, \beta_{n} \in(0, \infty)$ such that $z_{n}=\alpha_{n} x+\beta_{n} y$. Fix an arbitrary $w \in \overline{\mathcal{C}}(x, y)$. Then $L(w)<0$ and $w=\alpha_{w} x+\beta_{w} y$ for some $\alpha_{w}, \beta_{w} \in[0, \infty)$ with $\alpha_{w}+\beta_{w}>0$. Thus, taking an $s \in(0, \infty)$ with $1+s L(w)<0$, in view of (47), for sufficiently large $n \in \mathbb{N}$, we obtain

$z_{n}+g\left(z_{n}\right) s w=z_{n}-\frac{1}{n} s w=\left(\alpha_{n}-\frac{1}{n} s \alpha_{w}\right) x+\left(\beta_{n}-\frac{1}{n} s \beta_{w}\right) y \in \mathcal{C}(x, y) \subset \mathcal{C}$.

Hence, applying (6), we get

$$
f\left(z_{n}+g\left(z_{n}\right) s w\right)=f\left(z_{n}\right) f(s w) \text { for sufficiently large } n \in \mathbb{N} \text {. }
$$

Moreover, according to (47), we obtain

$1+L\left(z_{n}+g\left(z_{n}\right) s w\right)=1+L\left(z_{n}\right)-\frac{1}{n} s L(w)=-\frac{1}{n}(1+s L(w))>0$ for $n \in \mathbb{N}$.

Thus, making use of (43), we get

$$
f\left(z_{n}+g\left(z_{n}\right) s w\right)=\left(-\frac{1}{n}(1+s L(w))\right)^{r}>0 \text { for } n \in \mathbb{N} .
$$

On the other hand, in view of (46), $w \in \mathcal{C}_{(l)}$ for some $l \in\{4,5,6\}$. Hence, as $1+s L(w)<0$ and $1+L\left(z_{n}\right)=-\frac{1}{n}$ for $n \in \mathbb{N}$, applying Proposition 2.2, for every $n \in \mathbb{N}$, we obtain 


$$
f\left(z_{n}\right) f(s w)= \begin{cases}-\left|\frac{1}{n}(1+s L(w))\right|^{r} & \text { whenever } l \in\{4,5\}, l \neq k \\ 0 & \text { whenever } l=6 .\end{cases}
$$

Thus, taking into account (48) and (49), we get that $k=l$. Hence $w \in \mathcal{C}_{(k)}$. Since $w \in$ $\overline{\mathcal{C}}(x, y)$ is fixed arbitrarily, this implies that $\overline{\mathcal{C}}(x, y) \subseteq \mathcal{C}_{(k)}$. In particular $x, y \in \mathcal{C}_{(k)}$ and so $\mathcal{C}_{\left(j_{1}\right)} \cap \mathcal{C}_{(k)} \neq \emptyset$ and $\mathcal{C}_{\left(j_{2}\right)} \cap \mathcal{C}_{(k)} \neq \emptyset$. Thus, in view of (17), we get $j_{1}=k=j_{2}$, which yields a contradiction.

Case 2 Fix a $w \in \mathcal{C}(x, y)$. Then $L(w)<0$ and so, taking $w_{0}:=-\frac{w}{2 L(w)}$, we get $w_{0} \in \mathcal{C}(x, y)$ and $1+L\left(w_{0}\right)=\frac{1}{2}>0$. Hence, making use of (29) and (30), we obtain $f\left(w_{0}\right)=\frac{1}{2^{r}}$ and $g\left(w_{0}\right)=\frac{1}{2}$, respectively. Let $s \in(0, \infty)$ be such that $1+s L(w)<0$. Suppose that $j_{1} \in\{4,5\}$. Then, as $x \in \mathcal{C}_{\left(j_{1}\right)}$, according to Proposition 2.2, we have $f_{x}(s) \neq 0$. Moreover,

$$
w_{0}+g\left(w_{0}\right) s x=w_{0}+\frac{s}{2} x \in \mathcal{C}\left(x, w_{0}\right) \subset \mathcal{C}(x, y) \subseteq \mathcal{C}_{(6)} \subset \mathcal{C}
$$

Thus, in view of (6), we obtain

$$
f\left(w_{0}+g\left(w_{0}\right) s x\right)=f\left(w_{0}\right) f(s x)=\frac{1}{2^{r}} f_{x}(s) \neq 0 .
$$

On the other hand, we get

$$
1+L\left(w_{0}+g\left(w_{0}\right) s x\right)=1+L\left(w_{0}\right)+g\left(w_{0}\right) s L(x)=\frac{1}{2}(1+s L(x))<0,
$$

so making use of (29), we conclude that $f\left(w_{0}+g\left(w_{0}\right) s x\right)=0$. This is a contradiction. Consequently $j_{1}=6$. In the same way we obtain that $j_{2}=6$, which yields a contradiction.

Remark 3.1 From Theorems 2.1 and 3.1 it follows that if the functions $f, g: \mathcal{C} \rightarrow \mathbb{R}$ are continuous on rays, then the pair $(f, g)$ satisfies Eq. (6) if and only if there exist continuous functions $\tilde{f}, \tilde{g}: \mathbb{R} \rightarrow \mathbb{R}$ and a nontrivial linear functional $L:$ Lin $\mathcal{C} \rightarrow \mathbb{R}$ such that the pair $(\tilde{f}, \tilde{g})$ satisfies Eq. (4), $f=\tilde{f} \circ L_{\mid \mathcal{C}}$ and either $g=\tilde{g} \circ L_{\mid \mathcal{C}}$, or $L(\mathcal{C})=(-\infty, 0]$ and, for every $x \in \mathcal{C}, g(x)=(\tilde{g} \circ L)(x)$ whenever $1+L(x)>0$ and $g(x) \geq 0$, otherwise.

From Theorem 3.1 we derive the following result, which generalizes the main result in [19].

Corollary 3.1 Assume that a function $f: \mathcal{C} \rightarrow \mathbb{R}$ is continuous on rays. Then $f$ satisfies equation

$$
f(x+f(x) y)=f(x) f(y) \text { whenever } x, y, x+f(x) y \in \mathcal{C}
$$

if and only if $f=0$ or $f=1$ or there exists a nontrivial linear functional $L:$ Lin $\mathcal{C} \rightarrow$ $\mathbb{R}$ such that either $f(x)=1+L(x)$ for $x \in \mathcal{C}$, or $f(x)=\max \{1+L(x), 0\}$ for $x \in \mathcal{C}$. 
Corollary 3.2 If a function $f: \mathcal{C} \rightarrow \mathbb{R}$ is continuous on rays and satisfies Eq. (50), then there exists a function $F: X \rightarrow \mathbb{R}$ continuous on rays and satisfying equation

$$
F(x+F(x) y)=F(x) F(y) \text { for } x, y \in X
$$

such that $f(x)=F(x)$ for $x \in \mathcal{C}$.

Remark 3.2 The counterpart of Corollary 3.2 does not hold for Eq. (6) even in the case $X=\mathbb{R}$ and $\mathcal{C}=[0, \infty)$ (cf. [8]).

Now, we determine the solutions of Eq. (7).

Theorem 3.2 Assume that functions $F, G: X \rightarrow \mathbb{R}$ are continuous on rays. Let $G^{-}:=\{x \in \mathcal{C} \mid G(x)<0\}$ and $G^{-}-\mathcal{C}:=\left\{x-y \mid(x, y) \in G^{-} \times \mathcal{C}\right\}$. Then the pair $(F, G)$ satisfies Eq. (7) if and only if one of the following possibilities holds:

(i) $G$ is arbitrary and $F(x)=0$ for $x \in \mathcal{C} \cup\left(G^{-}-\mathcal{C}\right)$;

(ii) $G$ is arbitrary and $F(x)=1$ for $x \in \mathcal{C} \cup\left(G^{-}-\mathcal{C}\right)$;

(iii) $G(x)=1$ for $x \in \mathcal{C}$ and there exists a nontrivial linear functional $L:$ Lin $\mathcal{C} \rightarrow \mathbb{R}$ such that $F(x)=e^{L(x)}$ for $x \in \mathcal{C}$;

(iv) There exist an $r \in \mathbb{R} \backslash\{0\}$ and a linear functional $L:$ Lin $\mathcal{C} \rightarrow \mathbb{R}$ such that $r>0$ whenever $L(\mathcal{C}) \cap(-\infty, 0) \neq \emptyset$,

$$
G(x)=\max \{1+L(x), 0\} \text { for } x \in \mathcal{C}
$$

and

$$
F(x)=(\max \{1+L(x), 0\})^{r} \text { for } x \in \mathcal{C} ;
$$

(v) There exist an $r \in(0, \infty)$ and a linear functional $L:$ Lin $\mathcal{C} \rightarrow \mathbb{R}$ such that $L(\mathcal{C}) \cap(-\infty, 0) \neq \emptyset$,

$$
G(x)=1+L(x) \text { for } x \in \mathcal{C}
$$

and either

$$
F(x)=|1+L(x)|^{r} \quad \text { for } \quad x \in \operatorname{Lin} \mathcal{C}
$$

or

$$
F(x)=|1+L(x)|^{r} \operatorname{sgn}(1+L(x)) \text { for } x \in \operatorname{Lin} \mathcal{C} ;
$$

(vi) There exist an $r \in(0, \infty)$ and a linear functional $L:$ Lin $\mathcal{C} \rightarrow \mathbb{R}$ such that $L(\mathcal{C})=(-\infty, 0], F$ is of the form (52) and, for every $x \in \mathcal{C}$,

$$
G(x)=1+L(x) \text { whenever } 1+L(x)>0
$$

and $G(x) \geq 0$, otherwise.

Proof Assume that the pair $(F, G)$ satisfies (7). Then the functions $F_{\mid \mathcal{C}}$ and $G_{\mid \mathcal{C}}$ are continuous on rays and the pair $\left(F_{\mid \mathcal{C}}, G_{\mid \mathcal{C}}\right)$ satisfies Eq. (6). Hence, according to 
Theorem 3.1, one of the possibilities (a)-(f) holds. Note that (c) implies (iii), (d) yields (iv) and (f) leads to (vi).

If (a) holds then $F(x)=0$ for $x \in \mathcal{C}$. Moreover, if $G^{-} \neq \varnothing$ then taking a $z \in G^{-}-\mathcal{C}$, we have $z=u-y$ for some $u \in G^{-}$and $y \in \mathcal{C}$. Thus, as $-\frac{1}{G(u)} y \in \mathcal{C}$, making use of (7), we obtain

$$
F(z)=F(u-y)=F\left(u+G(u)\left(\frac{-1}{G(u)} y\right)\right)=F(u) F\left(\frac{-1}{G(u)} y\right)=0 .
$$

Hence $F(x)=0$ for $x \in G^{-}-\mathcal{C}$ and so (i) is valid. The similar arguments show that (b) leads to (ii).

Finally consider the case where (e) holds. Then (40) implies (53). Suppose that (41) holds. Then

$$
F(x)=|1+L(x)|^{r} \text { for } x \in \mathcal{C} .
$$

Furthermore, taking a $z \in \operatorname{Lin} \mathcal{C}$, we have $z=x-y$ for some $x, y \in \mathcal{C}$. If $1+L(x)<0$ then $-\frac{y}{1+L(x)} \in \mathcal{C}$ and so, applying (7), (53) and (56), we obtain

$$
\begin{aligned}
F(z) & =F(x-y)=F\left(x+(1+L(x))\left(\frac{-y}{1+L(x)}\right)\right) \\
& =F\left(x+G(x)\left(\frac{-y}{1+L(x)}\right)\right)=F(x) F\left(\frac{-y}{1+L(x)}\right) \\
& =|1+L(x)|^{r}\left|1-\frac{L(y)}{1+L(x)}\right|^{r}=|1+L(x)-L(y)|^{r} \\
& =|1+L(x-y)|^{r}=|1+L(z)|^{r} .
\end{aligned}
$$

If $1+L(x)=0$ then taking $x^{\prime}=2 x$ and $y^{\prime}=x+y$, we have $x^{\prime}, y^{\prime} \in \mathcal{C}, z=x-y=$ $x^{\prime}-y^{\prime}$ and $1+L\left(x^{\prime}\right)=1+2 L(x)=-1<0$. Therefore, arguing as in the previous case, we get that $F(z)=|1+L(z)|^{r}$. If $1+L(x)>0$ then taking an $x_{0} \in \mathcal{C}$ with $L\left(x_{0}\right)<0, \lambda:=-\frac{2}{L\left(x_{0}\right)}(1+L(x)), \tilde{x}:=x+\lambda x_{0}$ and $\tilde{y}:=y+\lambda x_{0}$, we get $\lambda>0$, $\tilde{x}, \tilde{y} \in \mathcal{C}, z=x-y=\tilde{x}-\tilde{y}$ and

$$
\begin{aligned}
1+L(\tilde{x}) & =1+L\left(x+\lambda x_{0}\right)=1+L(x)-\frac{2}{L\left(x_{0}\right)}(1+L(x)) L\left(x_{0}\right) \\
& =-(1+L(x))<0 .
\end{aligned}
$$

Therefore, making use of (7), (53) and (56), as previously we conclude that $F(z)=$ $|1+L(z)|^{r}$. In this way we have proved that (41) implies (54). The similar arguments show that (42) leads to (55). Thus, (v) is valid.

The converse is easy to check.

We conclude the paper with the result describing the solutions of the equation

$$
F(x+F(x) y)=F(x) F(y) \text { for } x, y \in \mathcal{C}
$$

in the class of functions $F: X \rightarrow \mathbb{R}$ continuous on rays. It generalizes the main result in [1]. 
Corollary 3.3 Assume that a function $F: X \rightarrow \mathbb{R}$ is continuous on rays. Then $F$ satisfies Eq. (57) if and only if one of the following possibilities holds:

(i) $F(x)=0$ for $x \in \mathcal{C}$;

(ii) $F(x)=1$ for $x \in \mathcal{C}$;

(iii) There exists a nontrivial linear functional $L: \operatorname{Lin} \mathcal{C} \rightarrow \mathbb{R} \operatorname{such} F(x)=\max \{1+$ $L(x), 0\}$ for $x \in \mathcal{C}$;

(iv) There exist a linear functional $L:$ Lin $\mathcal{C} \rightarrow \mathbb{R}$ such that $L(\mathcal{C}) \cap(-\infty, 0) \neq \emptyset$ and $F(x)=1+L(x)$ for $x \in \operatorname{Lin} \mathcal{C}$.

Acknowledgments The authors wish to express their thanks to the referee for valuable comments that helped to improve the layout of the results. The research was supported, in part, by ESF project CZ.1.07/2.3.00/20.0002. The work of the first author was partially supported by the Centre for Innovation and Transfer of Natural Sciences and Engineering Knowledge.

Open Access This article is distributed under the terms of the Creative Commons Attribution 4.0 International License (http://creativecommons.org/licenses/by/4.0/), which permits unrestricted use, distribution, and reproduction in any medium, provided you give appropriate credit to the original author(s) and the source, provide a link to the Creative Commons license, and indicate if changes were made.

\section{References}

1. Aczél, J., Schwaiger, J.: Continuous solutions of the Gołąb-Schinzel equation on the nonnegative reals and on related domains. Österr. Akad. Wiss. Math. Natur. Kl. Sitzungsber. II(208), 171-177 (1999)

2. Brzdęk, J.: On continuous solutions of a conditional Gołąb-Schinzel equation. Anz. Österreich. Akad. Wiss. Math.-Natur. K1. 138, 3-6 (2001)

3. Brzdęk, J.: The Gołąb-Schinzel equation and its generalizations. Aequationes Math. 70, 14-24 (2005)

4. Brzdęk, J., Mureńko, A.: On a conditional Gołąb-Schinzel equation. Arch. Math. (Basel) 85, 503-511 (2005)

5. Chudziak, J.: Semigroup-valued solutions of the Gołąb-Schinzel type functional equation. Abh. Math. Sem. Univ. Hamburg 76, 91-98 (2006)

6. Chudziak, J.: Semigroup-valued solutions of some composite equations. Aequationes Math. 88, 183198 (2014)

7. Chudziak, J.: Continuous on rays solutions of a Gołąb-Schinzel type equation. Bull. Aust. Math. Soc. 91, 273-277 (2015)

8. Chudziak, J., Kočan, Z.: Continuous solutions of conditional composite type functional equations. Results Math. 66, 199-211 (2014)

9. Jabłońska, E.: Continuous on rays solutions of an equation of the Gołąb-Schinzel type. J. Math. Anal. Appl. 375, 223-229 (2011)

10. Jabłońska, E.: The Pexiderized Gołąb-Schinzel functional equation. J. Math. Anal. Appl. 381, 565-572 (2011)

11. Jabłońska, E.: Remarks concerning the pexiderized Gołąb-Schinzel functional equation. J. Math. Appl. 35, 33-38 (2012)

12. Jabłońska, E.: On continuous solutions of an equation of the Gołąb-Schinzel type. Bull. Aust. Math. Soc. 87, 10-17 (2013)

13. Jabłońska, E.: On locally bounded above solutions of an equation of the Gołąb-Schinzel type. Aequationes Math. 87, 125-133 (2014)

14. Jabłońska, E.: On continuous on rays solutions of a composite-type equation. Aequationes Math. 89, 583-590 (2015)

15. Kahlig, P., Matkowski, J.: A modified Gołąb-Schinzel equation on restricted domain (with applications to meteorology and fluid mechanics). Österr. Akad. Wiss. Math.-Natur. Kl. Sitzungsber. II(211), 117136 (2002)

16. Kuczma M.: An Introduction to the Theory of Functional Equations and Inequalities. 2nd edn, Edited by Attila Gilányi. Birkhäuser, Berlin (2009)

17. Mureńko, A.: On solutions of the Gołąb-Schinzel equation. Int. J. Math. Math. Sci. 27, 541-546 (2001) 
18. Mureńko, A.: On solutions of a conditional generalization of the Gołąb-Schinzel equation. Publ. Math. Debrecen 63, 693-702 (2003)

19. Reich, L.: Über die stetigen Lösungen der Gołąb-Schinzel-Gleichung auf $\mathbb{R}$ und auf $\mathbb{R}_{\geq 0}$. Österr. Akad. Wiss. Math.-Natur. Kl. Sitzungsber. II(208), 165-170 (1999)

20. Reich, L.: Über die stetigen Lösungen der Gołąb-Schinzel-Gleichung auf $\mathbb{R}_{\geq 0}$. Anz. Österreich. Akad. Wiss. Math.-Natur. K1. 138, 7-12 (2001)

21. Sablik, M.: A conditional Gołąb-Schinzel equation. Anz. Österreich. Akad. Wiss. Math.-Natur. K1. 137, 11-15 (2000) 